2013

\title{
Ion Heating And Short Wavelength Fluctuations In A Helicon Plasma Source
}

\author{
E. E. Scime \\ J. Carr \\ M. Galante \\ R. M. Magee \\ R. Hardin
}

Follow this and additional works at: https://researchrepository.wvu.edu/faculty_publications

\section{Digital Commons Citation}

Scime, E. E.; Carr, J.; Galante, M.; Magee, R. M.; and Hardin, R., "Ion Heating And Short Wavelength Fluctuations In A Helicon Plasma Source" (2013). Faculty Scholarship. 570.

https://researchrepository.wvu.edu/faculty_publications/570 


\title{
Ion heating and short wavelength fluctuations in a helicon plasma source
}

\author{
E. E. Scime, ${ }^{1}$ J. Carr, Jr., ${ }^{1}$ M. Galante,${ }^{1}$ R. M. Magee, ${ }^{1}$ and R. Hardin ${ }^{2}$ \\ ${ }^{1}$ Department of Physics, West Virginia University, Morgantown, WV 26506, USA \\ ${ }^{2}$ Oak Ridge National Laboratory, Oak Ridge, TN 37831, USA
}

(Received 24 October 2012; accepted 12 February 2013; published online 4 March 2013)

\begin{abstract}
For typical helicon source parameters, the driving antenna can couple to two plasma modes; the weakly damped "helicon" wave, and the strongly damped, short wavelength, slow wave. Here, we present direct measurements, obtained with two different techniques, of few hundred kHz, short wavelength fluctuations that are parametrically driven by the primary antenna and localized to the edge of the plasma. The short wavelength fluctuations appear for plasma source parameters such that the driving frequency is approximately equal to the lower hybrid frequency. Measurements of the steady-state ion temperature and fluctuation amplitude radial profiles suggest that the anomalously high ion temperatures observed at the edge of helicon sources result from damping of the short wavelength fluctuations. Additional measurements of the time evolution of the ion temperature and fluctuation profiles in pulsed helicon source plasmas support the same conclusion. (C) 2013 American Institute of Physics. [http://dx.doi.org/10.1063/1.4794351]
\end{abstract}

\section{INTRODUCTION}

In our analysis of our previous experimental studies, we have suggested that the appearance of substantial ion heating at the specific combinations of driving antenna frequency and magnetic field strength that result in the equivalence of the driving antenna frequency with the lower hybrid frequency provided strong, but indirect, evidence of the damping of short wavelength, "slow" wave fluctuations in the edge of helicon sources (see Fig. 1). ${ }^{1,2}$ Consistent with this hypothesis was the measurement of hollow ion temperature profiles at those same helicon source parameters, i.e., perpendicular ion heating occurring preferentially in the plasma edge. What was missing from those studies was direct measurement of the existence of short wavelength fluctuations in the helicon source. For typical helicon source parameters, the driving antenna can couple to two plasma modes; the weakly damped, bounded whistler or fast "helicon" wave, and the strongly damped, slow or "Trivelpiece-Gould" (TG) wave. $^{3}$ Internal magnetic field measurements routinely demonstrate the existence of wave fields consistent with bounded whistler waves in helicon sources. ${ }^{4}$ However, measurement of the slow wave is considerably more difficult given its extremely short wavelength and evanescent nature for plasma densities typical of helicon sources, even in the lower densities at the radial edge of the plasma. Some researchers have attempted to identify the slow wave in the edge through direct current measurements ${ }^{5}$ or indirect methods such as comparison of antenna impedance measurements with theoretical predictions for slow wave coupling. ${ }^{6}$

Here, we present probe and coherent microwave scattering measurements of spatially localized, few hundred $\mathrm{kHz}$, short wavelength fluctuations in an argon helicon plasma. These measurements and similar results reported by the pulsed helicon source HE-L group at Ruhr-Universität ${ }^{7}$ demonstrate that excitation of short wavelength waves does occur in helicon sources. As in the Ruhr experiments, the short wavelength fluctuations appear to be parametrically driven sidebands that arise through coupling of the primary antenna to other lower hybrid and ion acoustic waves. Parametric excitation of waves in helicon sources has been observed in other helicon experiments. ${ }^{8-10}$ Because the waves observed in these experiments are at considerably lower frequencies than in the Ruhr experiments, the wave phases' velocities are much smaller and therefore the waves can directly interact with ions. In fact, these waves only appear at the same plasma source parameters for which the enhanced, edge localized, ion heating also occurs.

We begin this report with a description of the experimental apparatus and diagnostics followed by a very brief review of the salient features of the fast and slow waves and the relevant lower hybrid resonance features in the slow wave dispersion relation. We then present our fluctuation measurements and discuss them in light of the resonant ion heating and ion heating profile observations. Measurements of the time evolution of the ion temperature and fluctuation profiles, discussed next, provide additional confirmation of the ion heating due to wave damping hypothesis through observations of the development of hollow ion temperature profiles at the same time short wavelength fluctuations appear in the plasma edge.

\section{EXPERIMENTAL APPARATUS}

The Hot hELicon eXperiment (HELIX) ${ }^{11}$ is a typical linear helicon source with a hybrid stainless steel-Pyrex ${ }^{\mathrm{TM}}$ vacuum chamber (Fig. 2): the Pyrex chamber is $61 \mathrm{~cm}$ long and $10 \mathrm{~cm}$ in diameter, the stainless steel section is $91 \mathrm{~cm}$ long and $15 \mathrm{~cm}$ in diameter. The stainless steel chamber opens into a $1.8 \mathrm{~m}$ diameter, $4.4 \mathrm{~m}$ long diffusion chamber. A $19 \mathrm{~cm}$, $m=+1$ helical antenna is wrapped around the Pyrex tube and couples the rf power supplied from a $2 \mathrm{~kW}$, ENI 1000, rf amplifier into the plasma. The antenna system operates over a frequency range of 6-18 MHz. Ten water-cooled electromagnets provide a constant and nearly uniform magnetic field of $0-1400 \mathrm{G}$. The base pressure of the HELIX-diffusion 


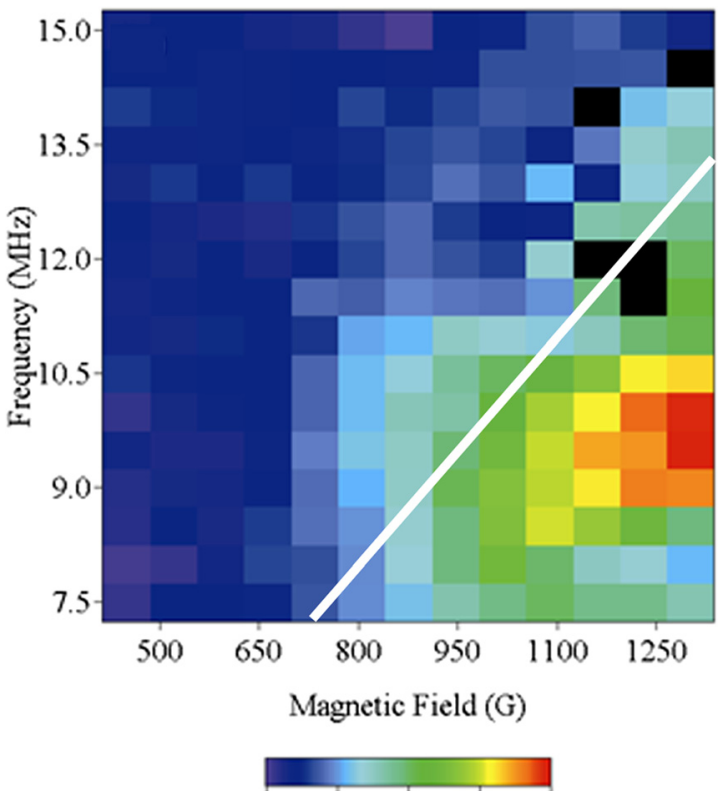

$\begin{array}{lllll}0.10 & 0.26 & 0.42 & 0.59 & 0.75\end{array}$

Ion Temperature (eV)

FIG. 1. Ion temperatures as a function of source frequency and magnetic field strength measured by laser induced fluorescence in HELIX for a neutral pressure of $6.7 \mathrm{mTorr}$ and an rf power of $750 \mathrm{~W}$. The white line denotes where the source frequency matches the calculated lower hybrid frequency along the axis of the source. At the plasma edge, the lower hybrid frequency curve shifts towards the lower right corner of the figure. The maximum ion temperature occurs for those source frequencies and magnetic fields at which the lower hybrid frequency matches the source frequency in the plasma edge. Reprinted with permission from J. L. Kline et al., Phys. Rev. Lett. 88, 195002 (2002). Copyright 2002 American Physical Society.

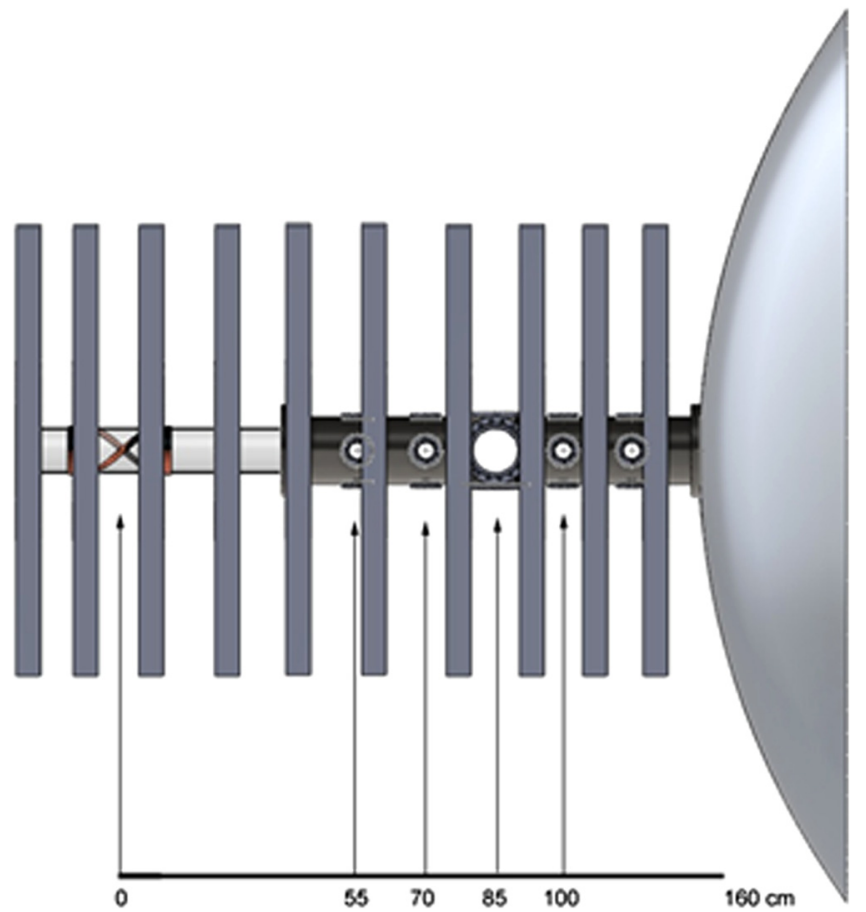

FIG. 2. Schematic of HELIX-expansion chamber apparatus (only a portion of the end of the expansion chamber is shown at the right of this figure). The $19 \mathrm{~cm}$ long $m=1$ antenna surrounds the glass portion of the chamber at $z=0 \mathrm{~cm}$. Ten electromagnets, aligned as shown, provide the magnetic field in the source region. The ports at 70,85 , and $100 \mathrm{~cm}$ were used for the $\mathrm{mm}$ wave and the electrostatic fluctuation measurements. chamber system is maintained at $10^{-7}$ Torr by three turbomolecular drag pumps. Typical electron temperatures and densities in the steady state-plasma are $T_{e} \approx 4 \mathrm{eV}$ and $n \sim 1$ $\times 10^{13} \mathrm{~cm}^{3}$ as measured with an rf compensated Langmuir probe. $^{12}$ All the measurements reported here were performed in the helicon source portion of the system. Additional descriptions of the experimental apparatus may be found in Refs. 13-15.

One significant change to the plasma source chamber for these experiments is the addition of a $300 \mathrm{GHz}$ coherent Thomson scattering (CTS) diagnostic designed to nonperturbatively measure the frequency and wavelength of electrostatic fluctuations. ${ }^{16}$ A schematic of the CTS geometry is shown in Fig. 3. The homodyne CTS scattering system, a quasi-optical interferometer, consists of three beam paths: An "interaction" beam path, a "scattered" beam path, and a "reference" beam path. As shown in Fig. 3, a flat mirror inside the vacuum chamber directs the scattered radiation from the fluctuations out of the chamber and onto the optical table. Rotating the mirror changes the angle of collection and thereby selects the wave number to be observed. The measurable scattered angles in HELIX range from $60^{\circ}$ to $90^{\circ}$, corresponding to fluctuation wave numbers of 63 to $89 \mathrm{rad} / \mathrm{cm}$ via the Bragg condition, $k=2 k_{0} \sin \left(\theta_{s} / 2\right)$, where $k_{0}$ is the incident wave number, $k$ is the fluctuation wave number, and $\theta_{s}$ is the scattering angle. ${ }^{14}$ The radial location of the collection volume depends on the angle and location of the collection mirror. Observable radii for $60^{\circ}$ scattering are from $r=-5$ to $-1.5 \mathrm{~cm}$, while for $90^{\circ}$ scattering, the observable radii range from -1.5 to $+5 \mathrm{~cm}$. Given our scattered power detection threshold of $750 \mathrm{pW}$, a density fluctuation amplitude of $5.5 \times 10^{11} \mathrm{~cm}^{-3}$ is required for observation of an electrostatic wave (a 5\% density fluctuation for a peak density of $10^{13} \mathrm{~cm}^{-3}$ ). Therefore, in the plasma edge, where the plasma density is typically one order of magnitude smaller, a $50 \%$ density fluctuation is required to detect waves with this diagnostic.

The electrostatic probe consists of two tungsten tips, $2 \mathrm{~mm}$ long and $0.33 \mathrm{~mm}$ in diameter. The tips are separated by a center to center distance of $0.61_{-0.13}^{+0.33} \mathrm{~mm}$. The upper

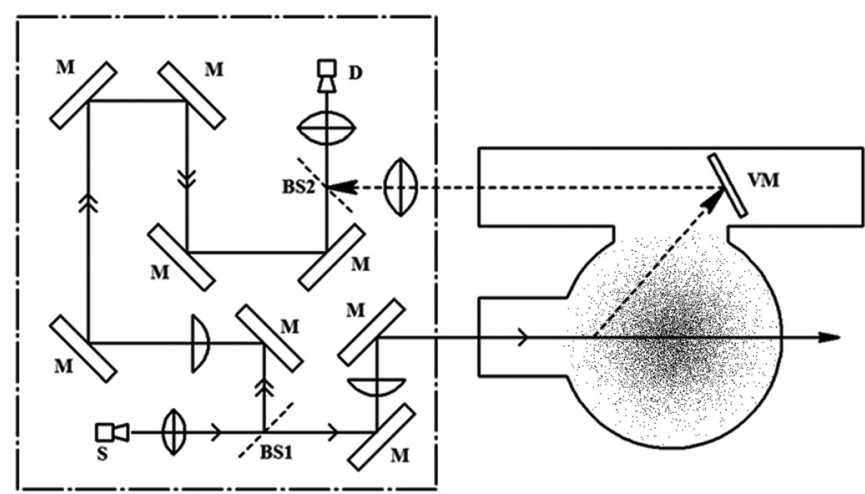

FIG. 3. Schematic of the mm-wave system and the optical path through a cross section of the helicon source (at $z=85 \mathrm{~cm}$ ): (S) is the mm-wave source, (D) is the detector, (M) are mirrors, (VM) is the adjustable vacuum mirror, (BS1) and (BS2) are the beam splitters. The cross sectional view includes the source chamber and additional structures that house the injection lens and collection mirror assembly. 
limit includes the center to center separation distance plus the individual radius of each tip, while the lower limit is based on the resolution limit of the method used to measure the tip spacing. The tips protrude from a 6-bore alumina shaft, $2 \mathrm{~mm}$ in outer diameter, and extending $7.6 \mathrm{~cm}$ from a boron nitride cap. For a maximum measured phase difference of $\pi$ and the nominal tip separation, the maximum measureable wave number is $\pm 51.5 \mathrm{rad} / \mathrm{cm}$. Typically, several time series of up to 262144 points with digitation rates up to a maximum of $100 \mathrm{MHz}$ are acquired for each probe tip. With such long time series, it is possible to subdivide the individual time series sets into smaller intervals to increase the number of effective data sets for averaging. The noise reduction obtained by creating subsets and increasing the total number of ensembles for averaging often outweighs the loss in frequency resolution. For the time series measurements in this work, the typical number of subdivisions used is 16 .

Analysis of the electrostatic probe measurements is accomplished by calculating the spectral density function (SDF) for each pair of probe tip measurements as a function of the angular frequency, $\omega$, and the wave number, $k$ (obtained from the phase difference measurements) ${ }^{17,18}$

$$
\begin{aligned}
S(n \Delta k, l \Delta \omega)= & \frac{1}{M} \sum_{j=1}^{M} I_{n \Delta k}\left[k^{(j)}(l \Delta \omega)\right] \\
& \times\left(\frac{1}{2}\left(P_{1}^{(j)}(l \Delta \omega)+P_{2}^{(j)}(l \Delta \omega)\right)\right),
\end{aligned}
$$

where $\Delta k$ and $\Delta \omega$ are the wave number and frequency bin widths, $l$ is the number of frequency increments, $n$ is the number of wave number increments, $M$ is the number of ensembles to average, $P_{1}(l \Delta \omega)$ and $P_{2}(l \Delta \omega)$ are the individual time series power spectra, and the selection function, $I_{n \Delta k}\left[k^{(j)}(l \Delta \omega)\right],{ }^{19}$ is

$$
\begin{aligned}
& I_{n \Delta k}\left[k^{(j)}(l \Delta \omega)\right] \\
& =\left[\begin{array}{cc}
1 & \left(n-\frac{1}{2}\right) \Delta k<k^{(j)}(l \Delta \omega)<\left(n+\frac{1}{2}\right) \Delta k \\
0 & \text { elsewhere }
\end{array}\right] .
\end{aligned}
$$

In essence, the SDF acts as a statistical histogram for the ensemble averaged spectral power contained in the individual power spectra. For each incremental frequency step and wave number, the spectral power is placed in the corresponding frequency-wave number bin. The result is a two dimensional array, comprised of the ensemble averaged spectral powers, for all frequencies and their associated wave numbers. A significant advantage of the SDF approach is the ability to identify several individual, or even a spectrum, of wave numbers associated with a given frequency. For all the spectral density plots in this work, the frequency resolution chosen is $\Delta f \sim 6103.5 \mathrm{~Hz}$ and the wave number resolution is $\Delta k \sim 0.68 \mathrm{rad} / \mathrm{cm}$. The wave number resolution is determined by $\Delta k=2 \pi /\left(d R_{\text {length }}\right)$, where $d$ is the probe tip separation and $R_{\text {length }}$ is the total number of wave number bins chosen for the SDF analysis. As a compromise between phase resolution and improved statistics, 151 wave number bins are used for the SDF analyses.
For laser induced fluorescence (LIF) measurements of the argon ion velocity distribution function (ivdf), we use the classic three-level LIF scheme in which the metastable $3 \mathrm{~d}^{2} \mathrm{G}_{9 / 2}$ state is optically pumped by $611.6616 \mathrm{~nm}$ (vacuum wavelength) radiation to the short living $4 \mathrm{p}^{2} \mathrm{~F}_{7 / 2}^{0}$ state, which then decays to the $4 s^{2} D_{5 / 2}$ state by emitting a photon at $461.09 \mathrm{~nm}$ (vacuum wavelength). Details of the laser used and the time resolved LIF diagnostic technique are given elsewhere. ${ }^{19}$ The injected laser light is modulated with an acousto-optic modulator (AOM) at $10 \mathrm{kHz}$ and then transported with a multimode, non-polarization preserving, optical fiber to the injection optics. Adequate signal-to-noise ratios of 10:1 or greater are obtained in pulsed plasmas by averaging the LIF signal at each laser wavelength over 200 or more plasma pulses. For these experiments, the time resolution is limited to $1 \mathrm{~ms}$ by the $1 \mathrm{~ms}$ integration time of the lock-in amplifier, needed to obtain sufficient photon counting statistics (10 on/off chopping cycles of the AOM). To obtain good precision velocity and temperature measurements, the ivdf is sampled at 128 equally spaced laser frequencies over a range of $20 \mathrm{GHz}$.

\section{FAST AND SLOW WAVES IN HELICON SOURCES}

Although researchers often turn to helicon sources for efficient plasma production and high densities, the exact mechanism responsible for coupling the rf power into the plasma is not completely understood. One of the first suggestions was that the rf coupling could be explained through either collisional damping of the helicon wave or Landau damping of the helicon wave on the electrons. ${ }^{20}$ Calculations determined that the collisional damping of the helicon wave, particularly for low neutral pressures, was insufficient to explain the power coupling. ${ }^{21}$ As for Landau damping of the helicon wave on the electrons, there needs to be enough of an energetic electron population for the damping to play a significant role. Measurements that hinted of energetic electron populations sufficient to contribute to the Landau damping process were reported, ${ }^{21-23}$ but subsequent measurements found that the population of energetic electrons was too sparse in the helicon source to make a significant contribution to the power deposition. It should be noted that in the same work, the measured resistive loading on the $\mathrm{rf}$ antenna was consistent with coupling to electrostatic waves in the plasma edge. Those measurements, which can be used as a proxy for the coupling efficiency of the antenna to the excitation of electrostatic waves, suggested that electrostatic waves were being excited and because of their short wavelength nature were strongly absorbed as they propagated inward.

Figure 4 shows a series of perpendicular wave number solutions for the cold plasma dispersion relation ${ }^{3}$ for the typical helicon source parameters described in the figure caption and assuming the parallel wavelength of the wave is comparable to the antenna length. For low magnetic fields, the real wave number solutions are identical. As the magnetic field strength increases, the solutions bifurcate into the fast wave and the slow wave. The perpendicular wave number of the fast helicon wave asymptotically approaches zero as the 


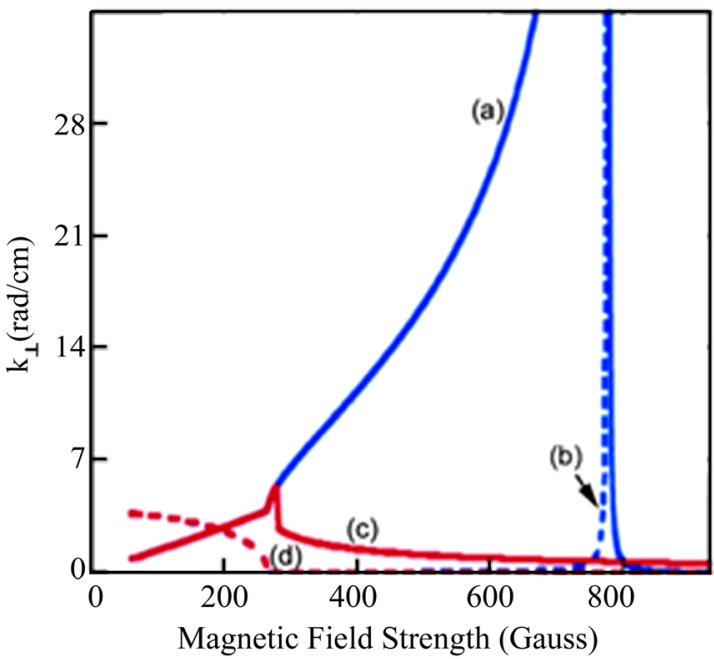

FIG. 4. Solutions of the cold plasma dispersion relation for $k_{\perp}$ with $n=5 \times 10^{12} \mathrm{~cm}^{-3}, k_{\|}=0.3 \mathrm{rad} / \mathrm{cm}, f=9 \mathrm{MHz}$, and no collisions. (a) The absolute value of the real $k_{\perp}$ for the slow wave, (b) the negative of the value of the imaginary $k_{\perp}$ for the slow wave, (c) the absolute value of the real $k_{\perp}$ for the fast wave, and (d) the negative of the value of the imaginary $k_{\perp}$ for the fast wave.

magnetic field increases. On the other hand, the perpendicular wave number of the slow wave solution continues to grow as the magnetic field is increased until the lower hybrid resonance is reached. The lower hybrid resonance occurs when $\varepsilon_{1}$ in the dispersion relation goes to zero, i.e., when the wave frequency $(\omega)$ is equal to $\omega_{L H}$. At $\omega=\omega_{L H}$, the perpendicular wave number is infinite in a collisionless plasma. The lower hybrid frequency,

$$
\frac{1}{\omega_{L H}^{2}}=\frac{1}{\omega_{c e} \omega_{c i}}+\frac{1}{\omega_{p i}^{2}+\omega_{c i}^{2}},
$$

depends on the ambient magnetic field strength and also the plasma density in low density plasma. The inclusion of collisions reduces the wave number at $\omega=\omega_{L H}$ to a finite number, while also slightly shifting the resonance frequency. The important physics that occurs at the resonance is that the wave becomes almost completely electrostatic $(\vec{k} \| \tilde{E})$ and the phase velocity, $V_{p}=\omega / k_{\perp}$, becomes small. If the phase velocity is comparable to the ion thermal speed, the slow wave could damp on the ions, thereby preferentially heating the ions in the perpendicular direction (these wavelengths are so small that the interaction length scales are a small fraction of an ion gyroradius). Note that the fast wave can play no direct role ion heating due to its small perpendicular wave number (large phase velocity) and lack of any resonance. Thus, any perpendicular ion heating, such as the heating shown in Figure 1, has to be attributed to the damping of the slow wave on the ions or another ion heating mechanism must be postulated.

The imaginary components of the wave number for both modes are also identical for low magnetic field strengths. Note that the negative of the imaginary components is shown in Figure 4, thus the waves, particularly the slow wave, are damped. The wave damping for the slow wave solution is a maximum at the lower hybrid resonance. In contrast, the damping of the fast wave is finite, but small across the lower hybrid resonance. Thus, the electrostatic slow wave, the TG wave ${ }^{24}$ could play a key role in the high rf absorption efficiency of helicon sources operating near the lower hybrid frequency. ${ }^{6,25-27}$

Several groups report calculations that shown that the rf power is absorbed more efficiently by the TG waves than by the helicon wave. ${ }^{28-33}$ Blackwell et al. reported experimental evidence for the TG mode in a helicon source through measurements of the parallel rf current. ${ }^{34}$ An important aspect of the measurements of Blackwell et al. is that the magnetic field was restricted to $25-60 \mathrm{G}$, while most helicon sources operate at magnetic fields in the hundreds of Gauss. With that in mind, their experiment with an rf driving frequency of $11 \mathrm{MHz}$, densities of $\sim 5 \times 10^{11} \mathrm{~cm}^{-3}$, and magnetic fields of $60 \mathrm{G}$, operated well above the lower hybrid frequency, where TG mode can still propagate but no lower hybrid resonance effects are expected.

The limited range of source driving frequencies available prevents most helicon source groups from exploring possible lower hybrid resonance effects and slow wave damping without drastically modifying the strength of the confining magnetic field. ${ }^{35,36}$ One of the key features of HELIX is the ability to continuously vary the driving frequency between 6 and $18 \mathrm{MHz}$, allowing for an extensive study of the lower hybrid resonance and the possible excitation of slow waves in argon plasmas without changing the particle confinement or cyclotron frequencies.

The challenge with measuring the slow wave, especially if the wave numbers are large enough to result in direct ionwave interactions, is that the expected wavelengths are on the order of $1 \mathrm{~mm}$ or smaller. Previous measurements of ionacoustic and lower hybrid waves with an electrostatic double probe in HELIX were limited to wave numbers $\leq 9.8 \mathrm{rad} / \mathrm{cm}$ $(\lambda \geq 6.4 \mathrm{~mm}) .{ }^{37}$ Measuring waves on the order of $1 \mathrm{~mm}$ or smaller are problematic with typical probes and therefore the CTS diagnostic was developed specifically for these experiments. CTS-like techniques have been employed in other helicon source experiments. Through measurements of enhanced microwave scattering off the upper hybrid resonance layer, $\omega_{U H}^{2} \equiv \omega_{p e}^{2}+\omega_{c e}^{2}$, both ion-acoustic and TG modes (lower hybrid waves) were observed propagating radially in a helicon plasma source. That diagnostic used two microwave sources at 9 and $28 \mathrm{GHz}$ with output powers of $50 \mathrm{~mW}$ and $20 \mathrm{~mW}$, respectively. ${ }^{42}$ Although the measurement indicated that both ion-acoustic and lower hybrid waves were present (with wave number magnitudes approaching $\sim 75 \mathrm{rad} / \mathrm{cm}$ ), the helicon source operated at a driving frequency of $13.56 \mathrm{MHz}$, where no significant lower hybrid resonance effects were expected.

\section{WAVE NUMBER AND ION TEMPERATURE MEASUREMENTS IN STEADY STATE PLASMAS}

The particular driving frequency and magnetic field strength combinations used in these experiments are indicated by black diamonds in Figure 5. Also shown in Figure 5 are the perpendicular wave numbers as a function of driving frequency and magnetic field strength predicted by the slow 


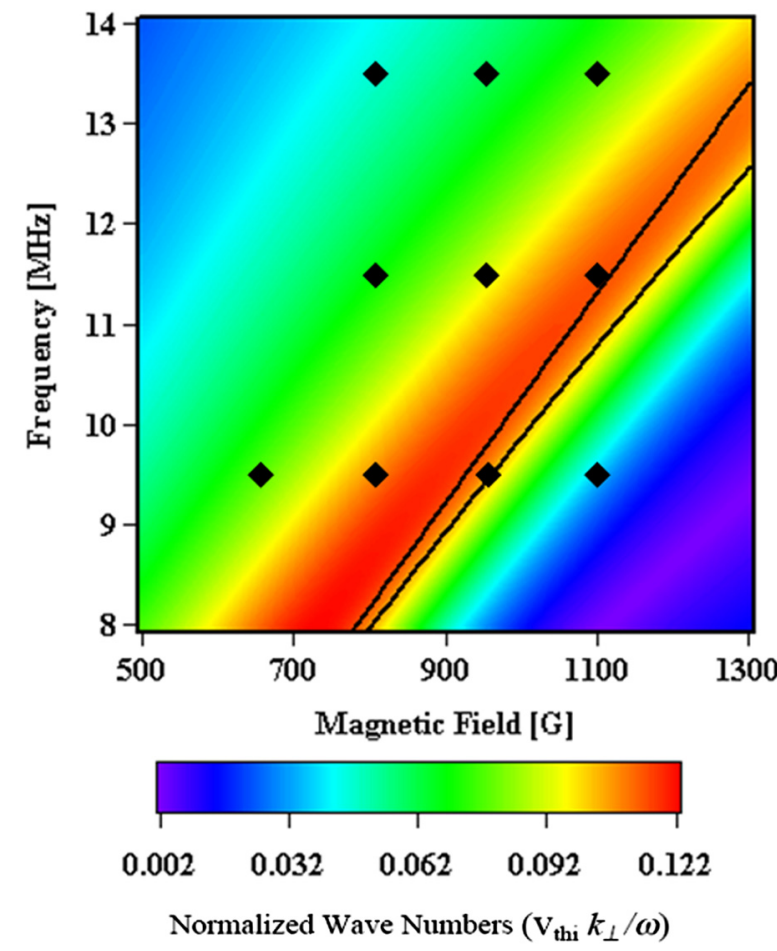

FIG. 5. Normalized perpendicular wave numbers for the slow wave calculated as a function of driving frequency and magnetic field strength for a neutral pressure of $8 \mathrm{mTorr}$ and an ion temperature of $0.2 \mathrm{eV}$. From left to right, the solid lines indicate for what frequency and magnetic field strength combinations the source frequency matches the lower hybrid frequency at $r=0 \mathrm{~cm}$ and at $r=5.5 \mathrm{~cm}$ (for typical helicon source density profiles). The black diamonds indicate the particular driving frequency and magnetic field strength combinations that were investigated in this work.

wave dispersion relation (Eq. (3)) for a neutral pressure of 8 mTorr, a perpendicular ion temperature of $0.2 \mathrm{eV}$, and for typical plasma density profiles in HELIX. The axial $(r=0)$ and edge $(r=5.5)$ lower hybrid frequencies are shown in Figure 5 as black curves to indicate the conditions at which the lower hybrid resonance would appear in the plasma at those radial locations. Because the specific experimental parameters of these experiments extend throughout the band of large normalized wave numbers, any observation of short wavelength fluctuations at the driving frequency with the electrostatic double probe or CTS system at these parameters would be consistent with excitation and damping of the slow wave near the lower hybrid resonance.

Based on the measured plasma density, the driving frequency normalized to the local lower hybrid frequency as a function of radius is shown in Figure 6 for each of the driving frequency and magnetic field strength combinations highlighted in Figure 5. For all driving frequencies and magnetic field strengths, the density profiles follow the typical radial profile observed in helicon plasma sources operating in the "helicon mode." ${ }^{38-40}$ Generally, the peak axial densities $(r=0 \mathrm{~cm})$ ranged from $1.5 \times 10^{13}$ to $3 \times 10^{13} \mathrm{~cm}^{-3}$ with densities of $\sim 1 \times 10^{12} \mathrm{~cm}^{-3}$ at the edge $(r=5.5 \mathrm{~cm})$. For a driving frequency of $9.5 \mathrm{MHz}$, the plasma source operates above the lower hybrid resonance frequency $\left(f / f_{\mathrm{LH}}>1\right)$ for magnetic field strengths $<800 \mathrm{G}$. At driving frequencies of 11.5 and $13.5 \mathrm{MHz}$, the source operates almost entirely in the condition $f / f_{\mathrm{LH}}>1$, except for a driving frequency of
11.5 MHz and magnetic field strength of $1100 \mathrm{G}$. Thus, the operational range of HELIX includes regimes above and below $f / f_{\mathrm{LH}}=1$. The radial density and electron temperature profiles for four different magnetic field strengths for three different $r f$ frequencies are shown in Figure 6. The most pronounced off-axis peaks in the electron temperature occur for a driving frequency of $9.5 \mathrm{MHz}$ and magnetic field strengths of 950 and $1100 \mathrm{G}$ - the same conditions expected to yield the largest slow wave perpendicular wave numbers. For example, compare the nearly flat electron temperature profile for a driving frequency of $13.5 \mathrm{MHz}$ and a magnetic field of $800 \mathrm{G}$ (red circles in Fig. 6(f)) and the profile obtained at a driving frequency of $9.5 \mathrm{MHz}$ and a magnetic field of $950 \mathrm{G}$ (the black squares in Fig. 6(d)). At the lower driving frequency and large magnetic fields (where the driving frequency nearly matches the lower hybrid frequency in the plasma edge as shown by the overlap between the black squares and the solid line in Fig. 6(a)), there is a distinct peak in the electron temperature at the edge of the plasma. Because particle and energy diffusion automatically leads to centrally peaked density and temperature profiles for either uniform or centrally peaked heating unless parallel losses dominate all the particle dynamics, even a flat electron temperature suggests some edge heating exists in the source. The appearance of a distinct electron temperature peak in the edge for only those cases, where the ratio of lower hybrid to driving frequencies equals unity (consider the overlap of the data points and the solid line in Figures 6(a)-6(c)) provides strong evidence for enhanced energy absorption at the lower hybrid resonance.

The perpendicular spectral density measured with the electrostatic probe at a radial location of $4.5 \mathrm{~cm}$ and for a neutral pressure of $8 \mathrm{mTorr}$, magnetic field of $800 \mathrm{G}$, driving frequency of $9.5 \mathrm{MHz}$, and $500 \mathrm{~W}$ of rf power is shown in Figure $7 \mathrm{for}$ frequencies above $200 \mathrm{kHz}$. There are three dominant spectral features in Figure 7, from $200 \mathrm{kHz}$ to $2 \mathrm{MHz}$ (low frequency (LF)), from 8 to $9.5 \mathrm{MHz}$ (low side band (LSB)), and from $9.5 \mathrm{MHz}$ to $11 \mathrm{MHz}$ (high side band (HSB)). Examining only the magnitude of the wave numbers for the moment, the most important detail is that all three features include fluctuations with wave numbers approaching the probe resolution limit of $\sim 51 \mathrm{rad} / \mathrm{cm}$. In fact, particularly for the LF wave, the wave numbers continue beyond the limit and phase wrap (due to the limit of $\pm \pi$ in the phase calculation) to the negative wave number side. Although no electromagnetic fluctuation measurements are made in this work to offer direct evidence that these fluctuations are purely electrostatic, the similarity to previous measurements in HELIX ${ }^{8}$ and the work of Kramer et al. ${ }^{41}$ strongly suggest that these fluctuations are purely electrostatic and are dominated by fluctuations in the electric potential and not in the electron temperature. Measurements obtained with the probe tips aligned along the magnetic field are shown in Figure 8. These data are representative of all the measurements obtained for parallel orientation of the probe tips; no significant spectral features at finite parallel wave numbers are observed. For these particular plasma conditions, there is a weak spectral feature at $\sim 1 \mathrm{MHz}$ that extends to parallel wave numbers of $\sim 10 \mathrm{rad} / \mathrm{cm}$. However, in general, the 

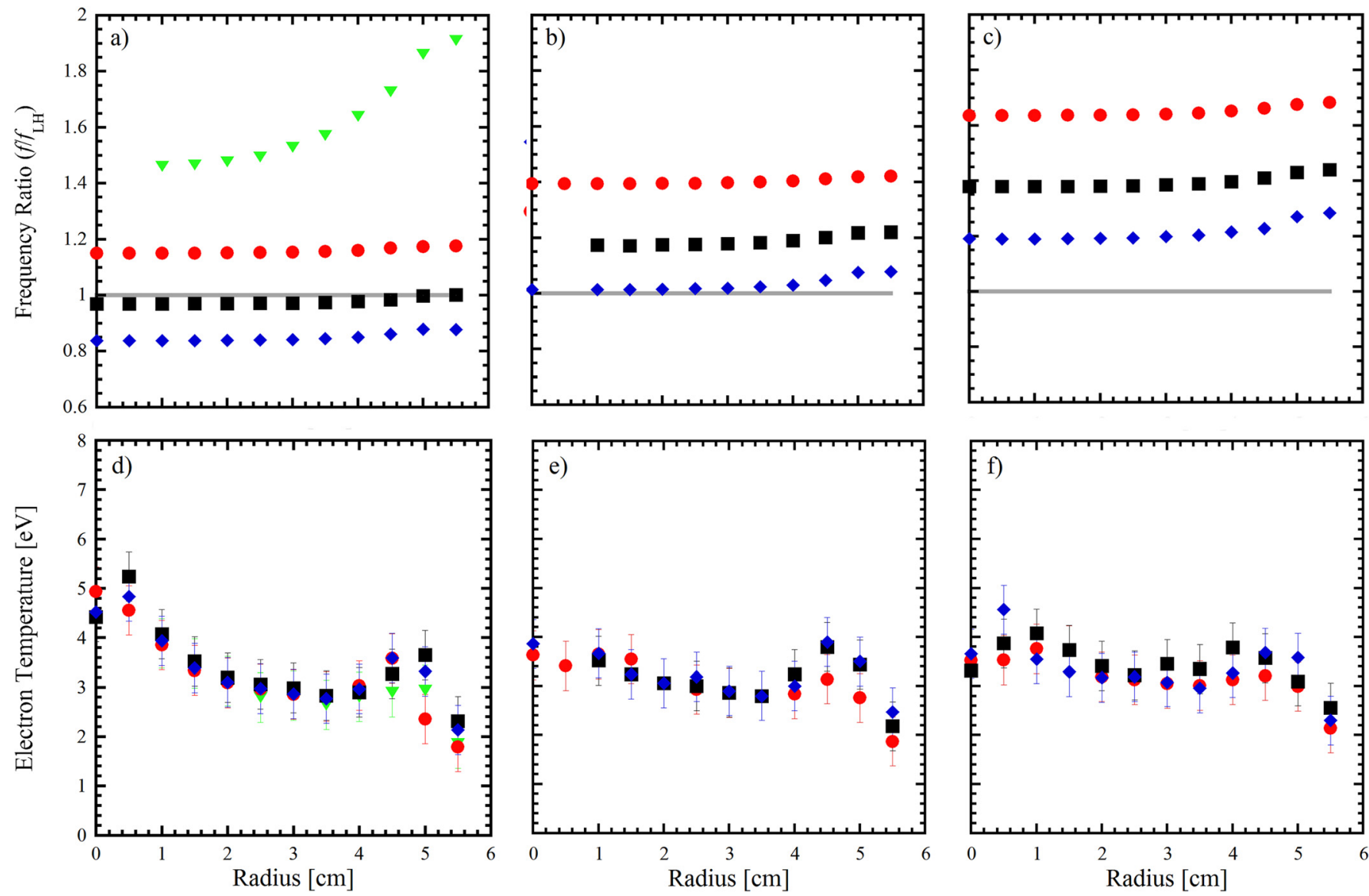

FIG. 6. Radial profiles of the ratio of the driving frequency relative to the lower hybrid frequency $\left(f / f_{\mathrm{LH}}\right)$, based on the measured density profiles, for driving

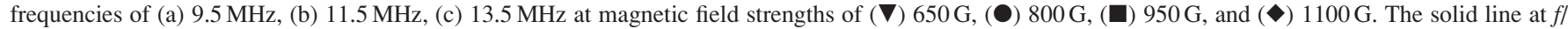
$f_{\mathrm{LH}}=1$ highlights the resonance condition. Electron temperature radial profiles for driving frequencies of (d) $9.5 \mathrm{MHz}$, (e) $11.5 \mathrm{MHz}$, and (f) $13.5 \mathrm{MHz}$ at the same magnetic fields.

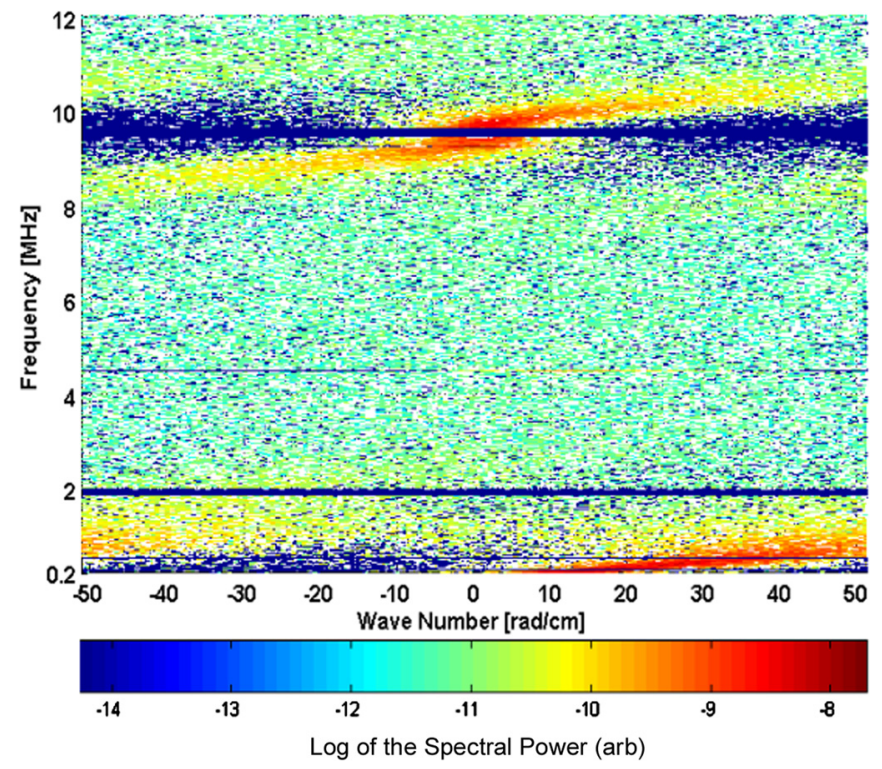

FIG. 7. Spectral density from the electrostatic double probe oriented perpendicular to the magnetic field at $r=4.5 \mathrm{~cm}$ for a magnetic field of $800 \mathrm{G}$, neutral pressure of $8 \mathrm{mTorr}$, and driving frequency of $9.5 \mathrm{MHz}$. A suppression window at $9.5 \pm 0.1 \mathrm{MHz}$ is applied to assist in identification of the fluctuations.

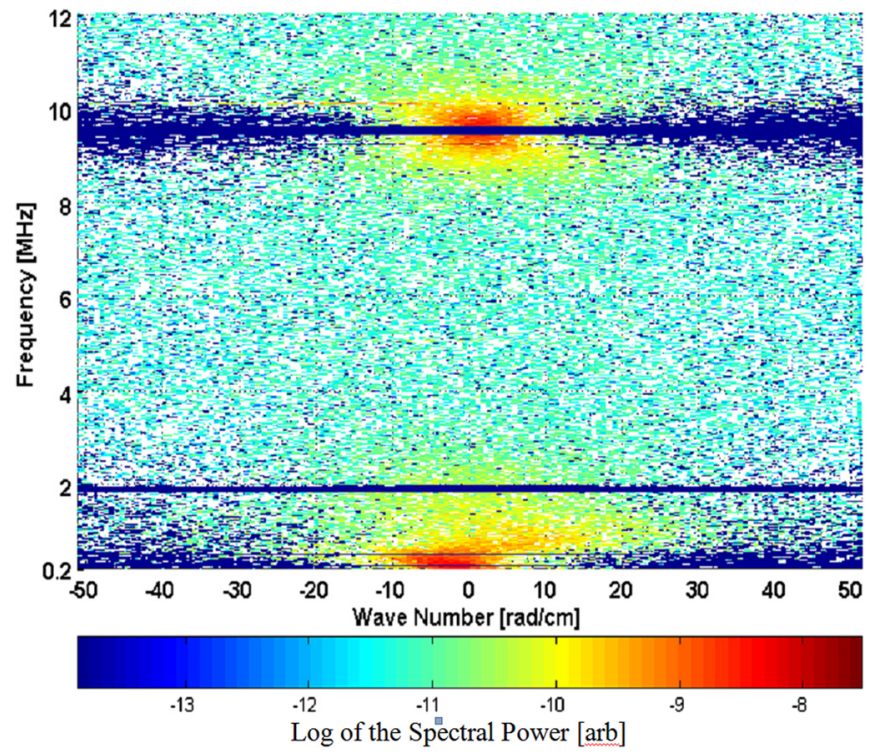

FIG. 8. Spectral density from the electrostatic double probe oriented parallel to the magnetic field at $r=4.5 \mathrm{~cm}$ for a magnetic field of $800 \mathrm{G}$, neutral pressure of $8 \mathrm{mTorr}$, and rf driving frequency of $9.5 \mathrm{MHz}$. A suppression window at $9.5 \pm 0.1 \mathrm{MHz}$ is applied to enhance the appearance of the fluctuations. 
observed fluctuations have no component of propagation along the background magnetic field, consistent with expectations for electrostatic ion cyclotron waves in a plasma with a large ion to electron mass ratio.

Because the fluctuations are propagating purely perpendicular to the magnetic field, the electrostatic probe can be rotated with respect to the magnetic field direction to reduce the effective separation of the probe tips and thereby increase the maximum measureable wave number (as shown in Figure 9). At an angle of $45^{\circ}$ relative to the axis of the source, the effective probe tip separation is approximately $0.043 \mathrm{~cm}$ and the maximum of the measureable perpendicular wave number range increases to $\pm 72.8 \mathrm{rad} / \mathrm{cm}$. The measureable wave number range is further extended by noticing that the structure in the fluctuation data shown in Figure 7 clearly continue past the $\pm \pi$ phase difference boundary and reappear with a $\pi$ offset on the other side of the figure. Thus, the "wrapped" portion of the spectrum is simply cut and pasted onto the correct end of the wave number spectrum. Based on Figure 9, fluctuations at $k_{\perp}$ $\sim 89 \mathrm{rad} / \mathrm{cm}$ are most likely to appear in the CTS measurements for $r=4.5 \mathrm{~cm}$ at frequencies $200 \mathrm{kHz}$ to $3 \mathrm{MHz}$ and from the $\mathrm{rf}$ driving frequency $(9.5 \mathrm{MHz})$ to approximately 12.5 MHz. If the CTS system was capable of detecting fluctuations at perpendicular wave numbers less than $40 \mathrm{rad} / \mathrm{cm}$, the chances of obtaining a measureable signal would be greatly increased. Note also that the wave number and frequency dependence of the broad spectrum of higher frequency fluctuation (LSB and USB waves) waves is nearly identical to that of the $(\sim 340 \mathrm{kHz})$ fluctuations.

The radial location of the peak in the low frequency $(\sim 340 \mathrm{kHz})$ fluctuation amplitude for the each of the ten sets of $\mathrm{rf}$ frequencies and magnetic field strengths specified in Figure 5 was determined by integrating the spectral density across a window of $55 \mathrm{kHz}$ centered on a frequency of $340 \mathrm{kHz}$ and also integrating over the perpendicular wave

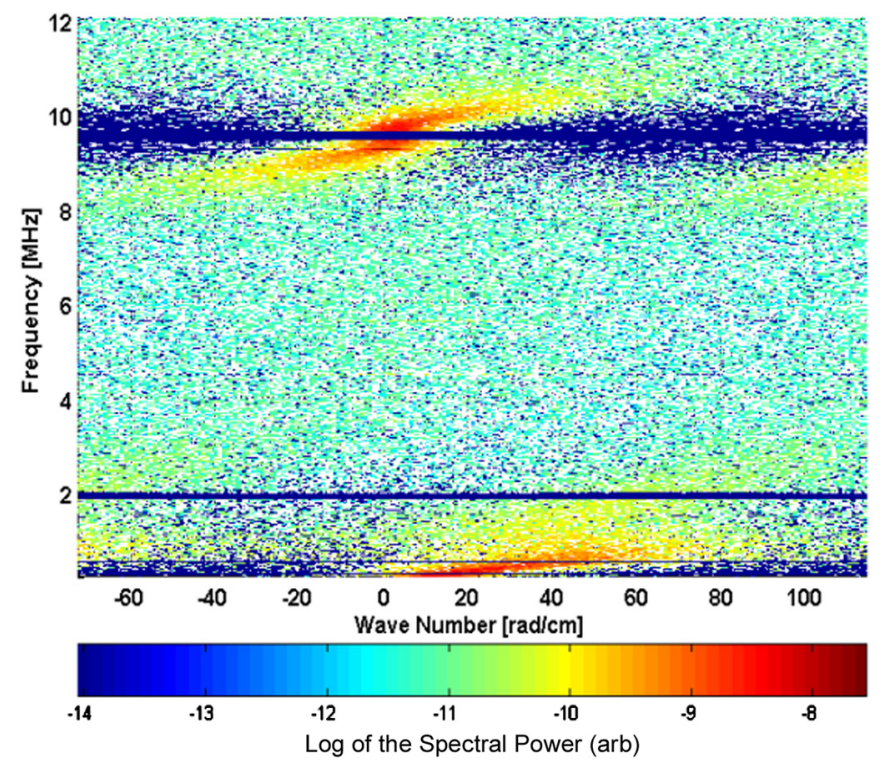

FIG. 9. Spectral density from the electrostatic double probe oriented $45^{\circ}$ to the magnetic field at $r=4.5 \mathrm{~cm}$ for a magnetic field of $800 \mathrm{G}$, neutral pressure of $8 \mathrm{mTorr}$, and driving frequency of $9.5 \mathrm{MHz}$. number range of 10 to $40 \mathrm{rad} / \mathrm{cm}$. The integrated values obtained with the probe tips aligned perpendicular to the equilibrium magnetic field direction are shown in Figure 10. For all source configurations, the $\sim 340 \mathrm{kHz}$ fluctuations are clearly localized to the plasma edge, $r \sim 4.5 \mathrm{~cm}$. At the lowest magnetic field strengths investigated $(650 \mathrm{G}$ and $800 \mathrm{G})$ at a driving frequency of $9.5 \mathrm{MHz}$ (Figure 10(a)), the fluctuations extended deeper into the plasma, about $r=2.5 \mathrm{~cm}$. The $r=4.5 \mathrm{~cm}$ location of the maximum in fluctuation amplitude corresponds to the same radial location at which increased electron temperatures were observed for the same plasma conditions.

CTS system scattering measurements (configured for wave numbers of $\sim 90 \mathrm{rad} / \mathrm{cm}$ ) were obtained for plasmas with a driving frequency of $9.5 \mathrm{MHz}$, a magnetic field strength of $800 \mathrm{G}$, and a neutral pressure of $8 \mathrm{mTorr}$. These parameters correspond to the conditions with the largest fluctuation amplitudes as measured with the electrostatic double probe. To maximize the signal-to-noise, each measurement was accomplished by acquiring 100 time series of 262144 points at a digitization rate of $100 \mathrm{MHz}$. The time series were then subdivided into 16 individual time series subsets before applying a Blackman window and then averaging the FFTs. By recording 100 individual time series and subdividing each time series into 16 subsets, the errors arising from normally distributed random noise were reduced by a factor of $\sim 1 / \sqrt{1600}$. Background measurements were obtained while the plasma source was on and the CTS collection optics window was blocked with a microwave absorbing material. The background power spectra were then subtracted from the actively sampled measurements. As discussed in detail in Ref. 42, the CTS system had an intrinsic, broadband, noise peak at $\sim 2.5 \mathrm{MHz}$ that prevented fluctuation measurements from 1 to $4 \mathrm{MHz}$. Unfortunately, the intrinsic noise peak was also modulated by $9.5 \mathrm{MHz}$ driving frequency. Because of sidebands created by the beating of the noise with the driving frequency, it was also impossible to distinguish any scattered signals at frequencies just above or below the driving frequency.

However, at frequencies on the order of $125 \mathrm{kHz}$ (much lower than the broadband noise peak), there was a significant difference in the background subtracted mixer-detector amplitudes. Shown in Figure 11 are CTS system scattering measurements, after background subtraction, integrated over the frequency band of $80 \mathrm{kHz}-150 \mathrm{kHz}$ versus radius. Also shown in Figure 11 are the electrostatic probe amplitudes for perpendicular wave numbers from 10 to $40 \mathrm{rad} / \mathrm{cm}$ integrated over a $55 \mathrm{kHz}$ band centered at $340 \mathrm{kHz}$ for the same plasma parameters and at the same radial locations. The similarity in radial profiles is striking and demonstrates that the CTS system successfully detected electrostatic fluctuations with wave numbers $\sim 90 \mathrm{rad} / \mathrm{cm}$ at frequencies $\sim 125 \mathrm{kHz}$.

For similar experimental parameters, ${ }^{8}$ the perpendicular ion thermal velocity, $V_{t h i}$, is $\sim 7 \times 10^{4} \mathrm{~cm} / \mathrm{s}$. The phase velocities of the waves observed by the CTS system, $f \sim 125 \mathrm{kHz}$ and $k_{\perp} \sim 89 \mathrm{rad} / \mathrm{cm}$, are much lower than the perpendicular ion thermal velocity, $V_{p} \sim 0.1 V_{t h i}$, and could therefore easily couple to the bulk of the ion distribution. The wavelength of these waves, $0.07 \mathrm{~cm}$, is so small that it is only $1 / 30$ th of the 

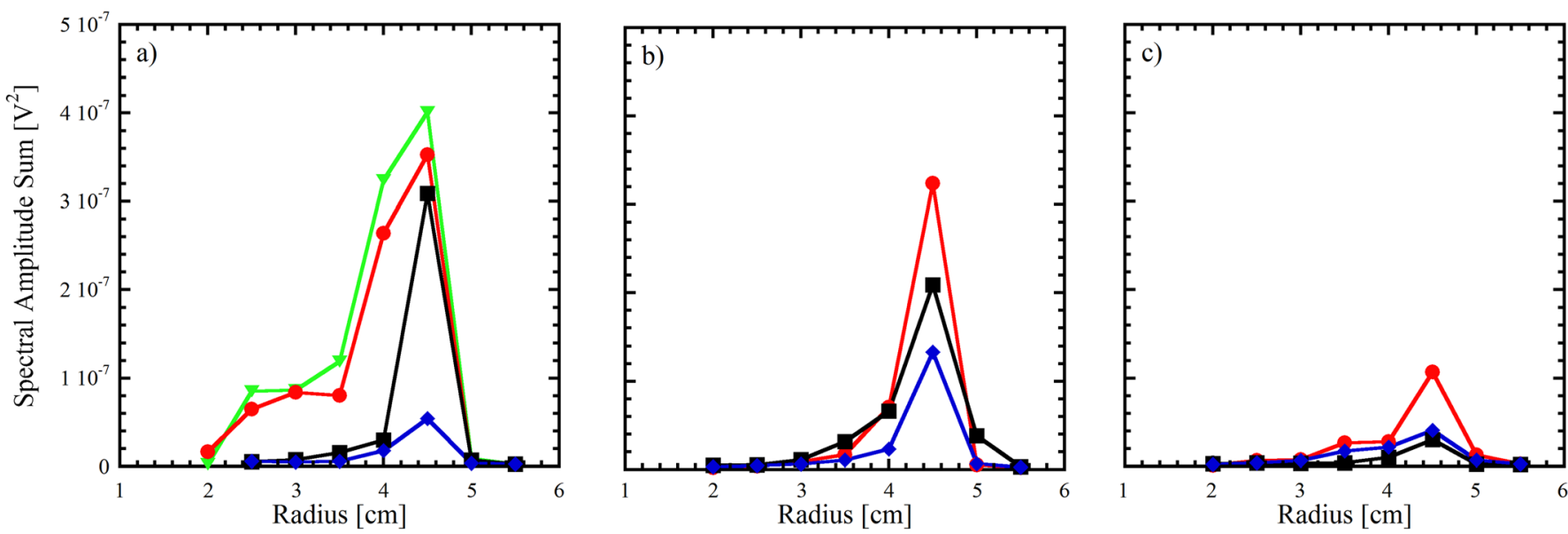

FIG. 10. Radial profiles of the average spectral density integrated over a $55 \mathrm{kHz}$ window centered at $340 \mathrm{kHz}$ and over the wave number range of 10 to $40 \mathrm{rad} /$

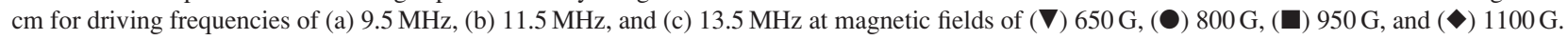

gyro circumference of the ions. In other words, ions can easily transit multiple wavelengths of the waves while maintaining their gyroorbit around the magnetic field lines.

\section{EVOLUTION OF ION TEMPERATURE AND FLUCTUATION PROFILES}

To investigate the possibility of a causal relationship between edge ion heating and electrostatic fluctuations in the edge of the helicon plasma, we investigated the time dependence of the radial profiles of the perpendicular ion temperature and the electrostatic fluctuation amplitude. For rf power pulses lasting $100 \mathrm{~ms}$, the initial $20 \mathrm{~ms}$ of the evolution of the radial profile of the ion temperature is shown in Figure 12(a). The magnetic field strength was $800 \mathrm{G}$, the driving frequency $9.5 \mathrm{MHz}$, the rf power $500 \mathrm{~W}$, and the neutral pressure was $8 \mathrm{mT}$ Torr. Prior to $2 \mathrm{~ms}$ into the discharge, the signal-to-noise is insufficient for a viable LIF measurement.

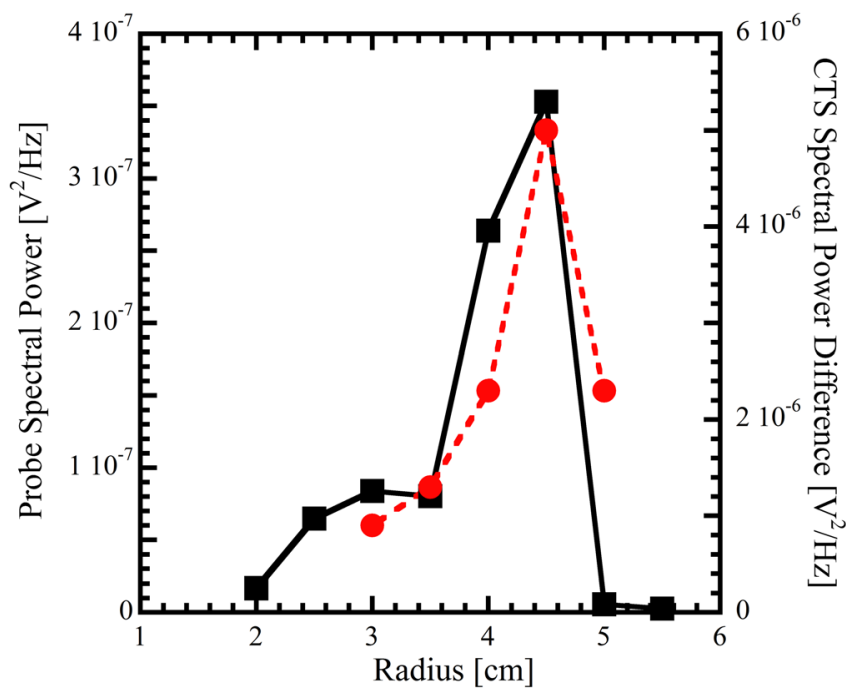

FIG. 11. Radial profiles of the integrated spectral power for the electrostatic double probe (squares) and the CTS diagnostic (circles) for a driving frequency of $9.5 \mathrm{MHz}$, a magnetic field strength of $800 \mathrm{G}$, and a neutral pressure of 8 mTorr. The electrostatic spectral power is integrated over $55 \mathrm{kHz}$ centered at $340 \mathrm{kHz}$ and the CTS spectral power is integrated over the range of $80-150 \mathrm{kHz}$.
The initial ion temperature profile measurements from 2 to $4 \mathrm{~ms}$ clearly show a centrally peaked profile. By $7 \mathrm{~ms}$ into the discharge, the core ion temperature has decreased and the edge ion temperature increased, resulting in an ion temperature profile that is clearly hollow. The hollow ion temperature profile persists throughout the remainder of the

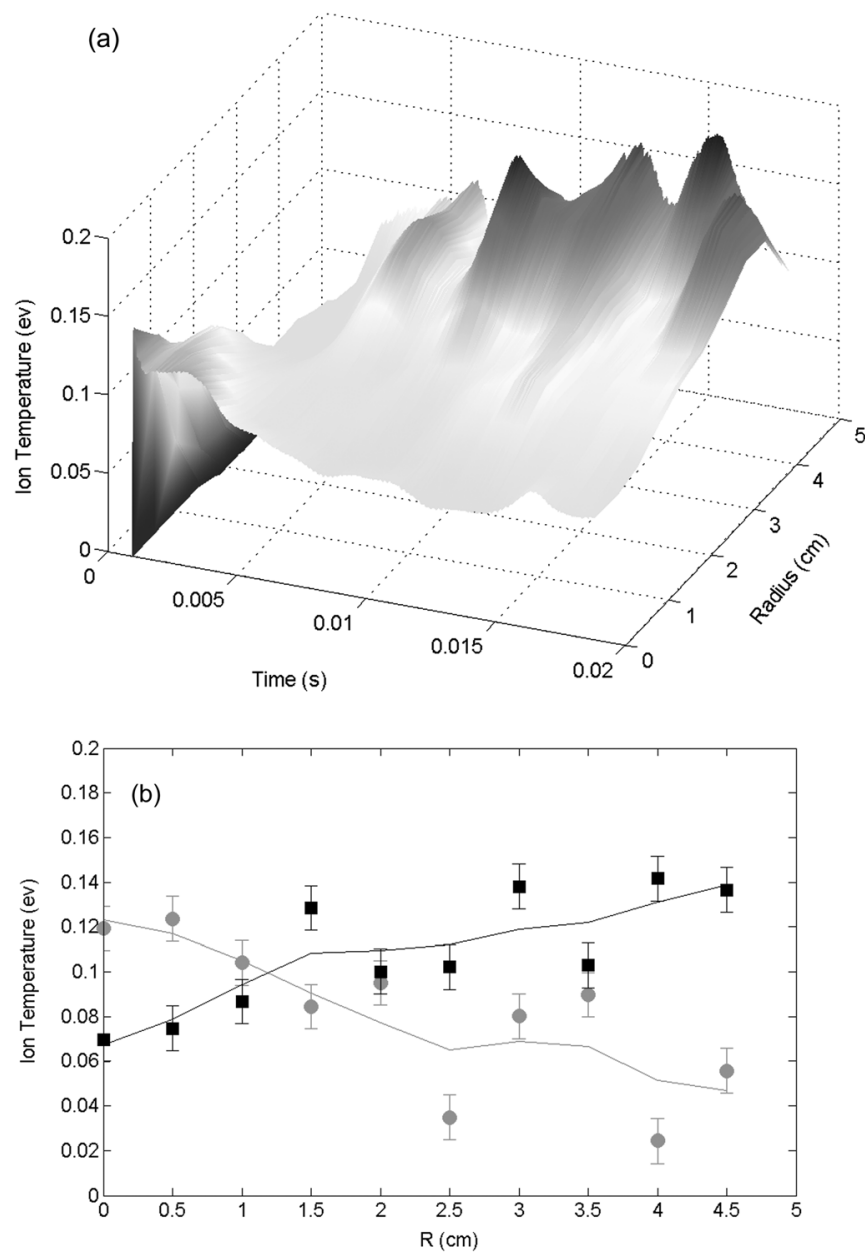

FIG. 12. (a) Radial profile versus time of the ion temperature during a $100 \mathrm{~ms}$ helicon source pulse. The first $20 \mathrm{~ms}$ of the discharge are shown. (b) Individual ion temperature profile measurements obtained at $2.7 \mathrm{~ms}$ (circles) and $14 \mathrm{~ms}$ (squares). 
$100 \mathrm{~ms}$ pulse. Shown in Figure 12(b) are individual ion temperature profile measurements acquired at $2.7 \mathrm{~ms}$ and $14 \mathrm{~ms}$ into the discharge. From $2.7 \mathrm{~ms}$ to $14 \mathrm{~ms}$, the core ion temperature drops by a factor of two and the edge ion temperature increases by a factor of three. The decrease in the core ion temperature and the increase in the edge ion temperature are both well outside the error bars of any individual measurement. The overall time-resolved ion temperature profile shown in Fig. 12(a) shows that there are fluctuations in the details of the profile shape over time, but that the basic structure of a peaked ion temperature profile that quickly becomes hollow persists throughout the entire discharge.

Electrostatic fluctuation measurements were simultaneously acquired at each radial location for a single tip from the electrostatic probe. The principal frequency components (essentially integrated over all wave numbers since the measurements are from a single probe tip) versus time of the fluctuations at $r=4.5 \mathrm{~cm}$ are shown in Figure 13(a). The time resolved frequency spectrum is obtained from a continuous wavelet transform ${ }^{43}$ of a single time series (a complex morlet function with large center frequency and broad bandwidth yielded sufficient frequency resolution given the $50 \mathrm{MHz}$ sampling rate of the time series measurement). In contrast to the continuous wavelet analysis method, the Fourier transform and spectral density function methods of fluctuation analysis required averages over many hundreds time series. In Figure 13(a), the driving wave dominates the frequency spectrum at $9.5 \mathrm{MHz}$. However, close inspection (Figure 13(b)) in the $100-300 \mathrm{kHz}$ range reveals multiple frequency peaks, the largest occurring at approximately $245 \mathrm{kHz}$. For purposes of comparison, the wavelet transform amplitude at $245 \mathrm{kHz}$ versus time is shown in Figure 14(c)

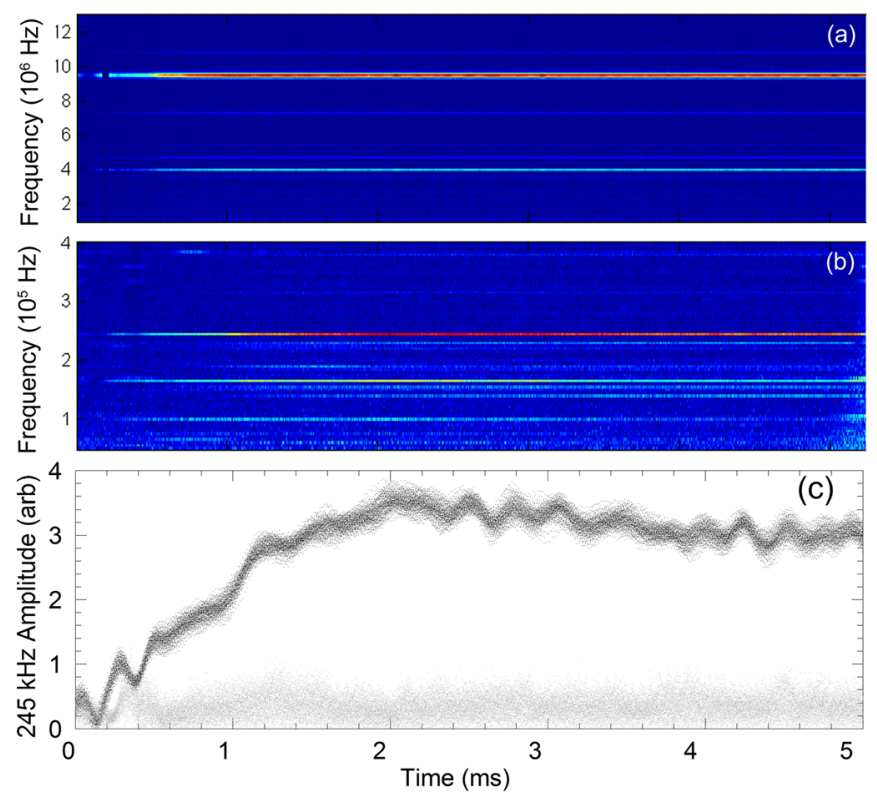

FIG. 13. (a) Continuous wavelet transform of the first $5 \mathrm{~ms}$ of the electrostatic fluctuations measured at a radial location of $3.5 \mathrm{~cm}$. The narrow spectral feature at $9.5 \mathrm{MHz}$ is the primary rf wave for the source. (b) Expanded view of the lower frequency portion of the spectrum shown in (a). The amplitude of the peak at $\sim 245 \mathrm{kHz}$ increases rapidly over the first $2 \mathrm{~ms}$ of the discharge. (c) The amplitude of the wavelet power spectrum at $245 \mathrm{kHz}$ for radial locations of $0 \mathrm{~cm}$ (light gray) and $3.5 \mathrm{~cm}$ (black).

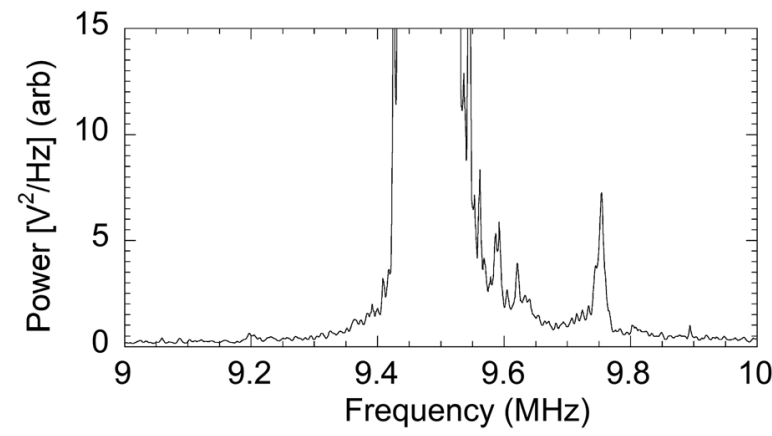

FIG. 14. Spectral power versus frequency acquired during a $5 \mathrm{~ms}$ wide sampling window $12.5 \mathrm{~ms}$ into the $100 \mathrm{~ms}$ pulsed discharge. The peak of the driving frequency has been cut off to highlight the sideband wave at 9.745 MHz.

for two radial locations. The continuous wavelet transform analysis provides, in contrast to a classic Fourier transform analysis, the time evolution of the frequency resolved fluctuation amplitude. The $245 \mathrm{kHz}$ fluctuation amplitude is substantially larger and increases in amplitude in a manner consistent with the temporal evolution of the edge ion temperature.

Although these measurements confirm that the temporal behavior of the edge ion temperature is consistent with the growth of few hundred kilohertz, short wavelength, waves in the plasma edge, they do not demonstrate the excitation of Trivelpiece-Gould waves by the source antenna. Evidence for the excitation of Trivelpiece-Gould waves appears in the details of the averaged fluctuation power spectrum measured during the pulsed discharge. Shown in Figure 14 is the averaged power spectrum (500 averages) based on a $5 \mathrm{~ms}$ wide acquisition window centered on $12.5 \mathrm{~ms}$ into the discharge at a radial location of $3.5 \mathrm{~cm}$. The primary driving frequency at $9.5 \mathrm{MHz}$ is evident as is a higher frequency, electrostatic sideband $245 \mathrm{kHz}$ above the driving frequency of the discharge. Another sideband wave at $125 \mathrm{kHz}$ above the driving frequency, the same frequency identified in the CTS measurements, is also visible in Figure 14. The higher frequency sidebands satisfy the frequency and wave number matching condition for parametric excitation by the primary rf wave in the helicon discharge. Thus, they are the link between the lower frequency waves seen in the probe and CTS measurements and the primary driving wave.

Examination of power spectra obtained from additional $5 \mathrm{~ms}$ windows centered on $2.5 \mathrm{~ms}, 7.5 \mathrm{~ms}$, and $17.5 \mathrm{~ms}$ into the pulse indicates that the upper sideband amplitude tracks the temporal evolution of the $245 \mathrm{kHz}$ wave amplitude during the initial $20 \mathrm{~ms}$ of the plasma pulse. As seen previously in this and other helicon sources, the sideband waves result from parametric decay of the intense driving wave. ${ }^{8,9}$ Therefore, the evolution of the ion heating in the plasma edge is well correlated with the growth of a short wavelength, $\sim 10 \mathrm{MHz}$, electrostatic wave localized to the edge of the plasma source, i.e., a Trivelpiece-Gould or "slow" wave.

\section{DISCUSSION}

The time resolved ion temperature profile measurements presented here demonstrate that the ions in helicon sources 
are preferentially heated at the edge of the source for source parameters consistent with the driving frequency matching the lower hybrid frequency in the plasma edge. In a pulsed helicon plasma, the ion temperature profile is initially centrally peaked, consistent with uniform or on-axis ion heating. As the amplitude of electrostatic waves driven parametrically by the primary rf wave increases, lower frequency electrostatic waves, at the sideband frequencies, appear and are spatially localized to the same region of the plasma at which the ion heating occurs. The temporal behavior of the wave amplitude mirrors that of the edge ion heating, i.e., the increases in edge ion heating and wave amplitude occur at the same time. The wave numbers of the lower frequency waves, measured by probes and a novel coherent Thomson scattering diagnostic operating at $300 \mathrm{GHz}$, are in excess of $90 \mathrm{~cm}^{-1}$. The frequency and wave number matching conditions for parametric decay of the driving wave, $k_{\mathrm{RF}}=k_{\mathrm{sb}}$ $+k_{\mathrm{lf}}$ and $f_{\mathrm{RF}}=f_{\mathrm{sb}}+f_{\mathrm{lf}}$ (where "RF" denotes the source frequency, "sb" denotes the sideband wave, and "lf" denotes the observed low frequency wave) indicate that waves exist at the pump frequency with wavelengths consistent with expectations for a heavily damped Trivelpiece-Gould wave. Therefore, these measurements not only provide strong evidence for the existence of Trivelpiece-Gould waves in helicon sources operated at the lower hybrid frequency but they also indicate a causal relationship between the TrivelpieceGould waves and the ion heating at the edge of the helicon source.

\section{ACKNOWLEDGMENTS}

This work was supported by NSF award PHY- 0902085. J.C., Jr. was supported by WV Space Grant and SREB graduate fellowships.

${ }^{1}$ J. L. Kline, E. E. Scime, R. F. Boivin, A. M. Keesee, and X. Sun, Plasma Sources Sci. Technol. 11, 413 (2002).

${ }^{2}$ J. L. Kline, E. E. Scime, R. F. Boivin, A. M. Keesee, X. Sun, and V. S. Mikhailenko, Phys. Rev. Lett. 88, 195002 (2002).

${ }^{3}$ S. Cho, Phys. Plasmas 7, 417 (2000).

${ }^{4}$ B. Davies, Plasma Phys. 11, 987 (1969); A. B. Jolly, G. Martelli, and J. F. Troughton, ibid. 11, 863 (1969).

${ }^{5}$ F. F. Chen and D. D. Blackwell, Phys. Rev. Lett. 82, 2677 (1999).

${ }^{6}$ K. P. Shamrai and V. B. Taranov, Plasma Sources Sci. Technol. 5, 474 (1996); D. Arnush, Phys. Plasmas 7, 3042 (2000).

${ }^{7}$ B. Lorenz, M. Kramer, V. I. Selenin, and Yu. M. Aliev, Plasma Sources Sci. Technol. 14, 623 (2005).

${ }^{8}$ J. L. Kline and E. Scime, Phys. Plasmas 10, 135 (2003).
${ }^{9}$ R. W. Boswell, O. Sutherland, C. Charles, J. P. Squire, F. R. Chang-Díaz, T. W. Glover, V. T. Jacobson, D. G. Chavers, R. D. Bengtson, E. A. Bering III, R. H. Goulding, and M. Light, Phys. Plasmas 11, 5125 (2004).

${ }^{10}$ F. F. Chen, IEEE Trans. Plasma Sci. 34, 718 (2006).

${ }^{11}$ X. Sun, C. Biloiu, R. Hardin, and E. Scime, Plasma Sources Sci. Technol. 13, 359 (2004).

${ }^{12}$ I. D. Sudit and F. F. Chen, Plasma Sources Sci. Technol. 3, 162 (1994).

${ }^{13}$ J. L. Kline, "Slow wave ion heating and parametric instabilities in the HELIX helicon source," Ph.D. dissertation (West Virginia University, Morgantown, 2002).

${ }^{14}$ A. M. Keesee, "Neutral density profiles in argon helicon plasmas," $\mathrm{Ph} . \mathrm{D}$ dissertation (West Virginia University, Morgantown, 2006).

${ }^{15}$ M. M. Balkey, "Optimization of a helicon plasma source for maximum density with minimal ion heating," Ph.D. dissertation (West Virginia University, Morgantown, 2000).

${ }^{16}$ R. Hardin and E. Scime, Rev. Sci. Instrum. 79, $10 \mathrm{E} 725$ (2008).

${ }^{17}$ J. M. Beall, Y. C. Kim, and E. J. Powers, J. Appl. Phys. 53, 3933 (1982).

${ }^{18} \mathrm{~S}$. Assadi, "Measurement of magnetic turbulence structure and nonlinear mode coupling of tearing fluctuations in the Madison symmetric torus reversed field pinch," Ph.D. dissertation (University of Wisconsin-Madison, Madison, 1994).

${ }^{19}$ I. A. Biloiu, X. Sun, and E. E. Scime, Rev. Sci. Instrum. 77, 10F301 (2006).

${ }^{20}$ F. F. Chen, Plasma Phys. Controlled Fusion 33, 339 (1991).

${ }^{21}$ P. Zhu and R. W. Boswell, J. Appl. Phys. 68, 1981 (1990).

${ }^{22}$ A. R. Ellingboe, R. W. Boswell, J. P. Booth, and N. Sadeghi, Phys. Plasmas 2, 1807 (1995).

${ }^{23}$ R. T. S. Chen and N. Hershkowitz, Phys. Rev. Lett. 80, 4677 (1998).

${ }^{24}$ A. W. Trivelpiece and R. W. Gould, J. Appl. Phys. 30, 1784 (1959).

${ }^{25}$ K. P. Shamrai and V. B. Taranov, Phys. Lett. A 204, 139 (1995).

${ }^{26}$ G. G. Borg and R. W. Boswell, Phys. Plasmas 5, 564 (1998).

${ }^{27}$ D. Arnush, Phys. Plasmas 7, 3042 (2000).

${ }^{28}$ I. V. Kamenski and G. G. Borg, Comput. Phys. Commun. 113, 10 (1998).

${ }^{29}$ T. Enk and M. Kramer, Phys. Plasmas 7, 4308 (2000).

${ }^{30}$ Y. Mouzouris and J. E. Scharer, Phys. Plasmas 5, 4253 (1998).

${ }^{31}$ B. H. Park, N. S. Yoon, and D. I. Choi, IEEE Trans. Plasma Sci. 29, 502 (2001).

${ }^{32}$ K. P. Shamrai and S. Shinohara, Phys. Plasmas 8, 4659 (2001).

${ }^{33}$ A. Ganguli, B. B. Sahu, and R. D. Tarey, Phys. Plasmas 14, 113503 (2007).

${ }^{34}$ D. D. Blackwell, T. G. Madziwa, D. Arnush, and F. F. Chen, Phys. Rev. Lett. 88, 145002 (2002).

${ }^{35}$ F. F. Chen, J. Vac. Sci. Technol. A 10, 1389 (1992).

${ }^{36}$ S. Yun, J. H. Kim, and H. Y. Chang, J. Vac. Sci. Technol. A 15, 673 (1997).

${ }^{37}$ J. L. Kline and E. E. Scime, Phys. Plasmas 10, 2127 (2003).

${ }^{38}$ M. J. Burin, G. R. Tynan, G. Y. Antar, N. A. Crocker, and C. Holland, Phys. Plasmas 12, 052320 (2005).

${ }^{39}$ C. Schroder, O. Grulke, T. Klinger, and V. Naulin, Phys. Plasmas 12, 042103 (2005).

${ }^{40}$ M. Light and F. F. Chen, Phys. Plasmas 2, 1084 (1995).

${ }^{41}$ M. Kramer, Y. M. Aliev, A. B. Altukhov, A. D. Gurchenko, E. Z. Gusakov, and K. Niemi, Plasma Phys. Controlled Fusion 49, A167 (2007).

${ }^{42}$ R. Hardin, Ph.D. dissertation, Department of Physics, West Virginia University, 2008.

${ }^{43}$ D. Jordan, R. W. Miksad, E. J. Powers, Rev. Sci. Instrum. 68, 1484 (1997). 